\title{
Fast and sensitive time-multiplexed gas sensing of multiple lines using a miniature telecom diode laser between $1529 \mathrm{~nm}$ and $1565 \mathrm{~nm}$
}

\author{
M. Lewander • A. Fried • P. Weibring • D. Richter • \\ S. Spuler • L. Rippe
}

Received: 2 November 2010 / Revised version: 11 April 2011 / Published online: 8 June 2011

(C) Springer-Verlag 2011

\begin{abstract}
High-sensitive multi-species detection around $1550 \mathrm{~nm}$ using a modulated grating Y-branch, MG-Y, diode laser tunable between $1529 \mathrm{~nm}$ and $1565 \mathrm{~nm}$ is presented. The MG-Y diode laser is based on the Vernier effect of two modulated gratings, and exhibits quasi-continuous tuning over $36 \mathrm{~nm}$. Multi-species detection is achieved by fast sequential scanning of single absorption lines of $\mathrm{CH}_{4}, \mathrm{CO}$, $\mathrm{C}_{2} \mathrm{H}_{2}$, and $\mathrm{CO}_{2}$ distributed over the tuning range of the diode laser. The laser wavelength is scanned about $10 \mathrm{GHz}$ around each absorption line for $5 \mathrm{~ms}$ and this is followed by a discrete large jump in operating wavelength to the next line.

The MG-Y diode laser has a good repeatability in output frequency between sequential scan segments $(<10 \mathrm{MHz})$ enabling averaging of the scans. The setup employs digital wavelength modulation spectroscopy, dWMS, with Fourier-
\end{abstract}

M. Lewander $\cdot$ A. Fried $\cdot$ P. Weibring $\cdot$ D. Richter $\cdot$ S. Spuler

L. Rippe

National Center for Atmospheric Research,

Earth Observing Laboratory, 3450 Mitchell Lane, Boulder, CO 80301, USA

A. Fried

e-mail: fried@ucar.edu

L. Rippe

e-mail: rippe@ucar.edu

M. Lewander ( $\varangle)$

Department of Physics, Atomic Physics Division,

Lund University, P.O. Box 118, 22100 Lund, Sweden

e-mail: marta.lewander@fysik.lth.se

M. Lewander · L. Rippe

Advanced Study Program, National Center

for Atmospheric Research, 3450 Mitchell Lane, Boulder,

CO 80301, USA based WMS retrieval, applicable through the use of data acquisition cards and coherent sampling.

Absorbance sensitivity using $2 f$-WMS signals of $7 \times$ $10^{-7}$ in $30 \mathrm{~s}$ (all precisions in this paper are at the $1 \sigma$ level) is demonstrated for sensing of four gases and $2 \times 10^{-7}$ when only one gas is detected. Corresponding numbers for $0.5 \mathrm{~s}$ of averaging are $4 \times 10^{-6}$ and $1 \times 10^{-6}$.

\section{Introduction}

Diode lasers were early recognized as suitable light sources for gas-absorption spectroscopy due to their reliability, compact size, and wavelength tunability, and are today a natural component in many gas sensors [1-3]. High sensitivity and selectivity of tunable diode laser absorption spectroscopy, TDLAS, makes it a fast chemical analysis tool which is used both in industry [4] and in various research fields [5]. The measured parameters include temperature, pressure, flow, and concentration of interesting gases, such as $\mathrm{O}_{2}, \mathrm{CH}_{4}$, $\mathrm{H}_{2} \mathrm{O}, \mathrm{CO}$, and $\mathrm{CO}_{2}$ [4]. One major limitation with the TDLAS technique is the inherent tuning range of the diode laser used, generally restricting the scanning bandwidth of the instrument to single-gas detection.

The progress of the gas sensing TDLAS technique is aided by the development of semiconductor lasers for telecommunication. The distributed feedback diode laser (DFB) and the vertical cavity surface emitting laser (VCSEL) are two among other telecommunication lasers which possess good spectroscopic characteristics such as tunability, single-mode operation, and high side mode suppression ratio (SMSR). However, the desirable aspect of multi-species detection is restricted by the tunability of these diode lasers, to typically less than a nm for a current-tuned DFB [6]. Multiplexing of several diode lasers, either in time or in frequency, allows 
additional sensing wavelengths, but increases the complexity and the cost of the system. Broad-band detection with a single diode laser is thus an attractive solution, but demands a larger tunability.

Within the telecommunication field the limited tuning range of diode lasers is also a concern, and large efforts have been made to overcome these issues [7, 8]. An example of such a widely tunable diode laser solution is the fabrication of a monolithical array of parallel individually controllable diode lasers [9]. For spectroscopy, such an array of quantum cascade DFB diode lasers, ranging from 8.7-9.4 $\mu \mathrm{m}$, has been demonstrated [10].

Another possibility to expand the tuning range of a diode laser is to create an external cavity. However, mechanically tuned gratings do not allow repeatable diode laser control and the stability of such mechanical tuning becomes an issue in field applications, particularly on highly vibrating platforms. Efforts have been invested into incorporating the grating directly on the laser chip. Examples of such a construction is the use of MEMS, micro-electromechanicalsystem, enhancing the tunability of a VCSEL to $60 \mathrm{~nm}$ [11].

Another approach to attain large tunability with one single diode laser is to use the Vernier effect, i.e. two differently spaced scales that together give a higher precision. Superimposing two fiber-coupled feedback reflection combs with different peak separations and seeding them into the diode laser allows large tunability by the combination. Possible reflectors for this approach are Fabry-Pérot cavities or gratings. Use of the Vernier effect with grating structures like sampled gratings, SG, superstructures gratings, SSG, and binary superimposed gratings, BSG, were first demonstrated on distributed Bragg reflectors, DBR, diode lasers and later on DFB diode lasers [7, 12]. These multi-section diode lasers are somewhat more complex to control and tune, but possess the ability to be more broadly tuned, typically $30 \mathrm{~nm}$ [6]. A couple of different placement configurations of the two grating sections exist. In the most common configuration the two gratings are positioned on each side of the gain medium of the diode laser with a phase section in between. Gas sensing, of water and hydrogen chloride at $1.8 \mu \mathrm{m}$, based on this approach has been shown for a DFB diode laser with a BSG with a tunability of $21 \mathrm{~nm}$ [12]. An alternative placement of the reflectors is in a fiber-coupled Yconfiguration, and is employed in the modulated grating Ybranch (MG-Y) diode laser investigated in this work. This laser was initially developed for telecom applications [13] but has already shown potential for spectroscopy $[6,14]$. In addition to the large tuning range other favorable spectroscopic characteristics are high SMSR ( $>40 \mathrm{~dB}$ ), rapid tunability, and vibration insensitivity. The MG-Y diode lasers and other Vernier tuned diode lasers also exhibit independent power and wavelength control. This results in that wavelength tunability is accomplished without large power changes allowing frequency modulation (FM) and scanning to be accomplished without large unwanted amplitude modulation (AM), referred to as residual amplitude modulation (RAM). In conventional diode lasers current and/or temperature tuning not only changes the output frequency but also the optical power. Scanning and modulation of such lasers can thus cause large excessive optical background, structure which can be further exacerbated by optical components, such as optical fiber amplifiers [15]. The characteristics of the MG-Y diode laser make it a suitable laser source for TDLAS measurements. We investigate in this study the MG-Y diode laser for highly sensitive multi-species detection. This is accomplished by rapidly scanning four absorption lines of trace gases $\left(\mathrm{CO}_{2}, \mathrm{CO}, \mathrm{CH}_{4}\right.$, and $\left.\mathrm{C}_{2} \mathrm{H}_{2}\right)$ separated by $30 \mathrm{~nm}$ in the $1550 \mathrm{~nm}$ wavelength region.

\section{Technical description}

\subsection{The MG-Y diode laser}

The modulated grating Y-branch diode laser (S3500 Syntune) is a diode laser with two integrated gratings in a Y-formation (Fig. 1), with an output power of $20 \mathrm{~mW}$. The reflections of the gratings are slightly different with respect to the peak separation, allowing the use of the Vernier effect. Combining the peaks from the two gratings expands the operating wavelength region of the diode laser to $30 \mathrm{~nm}$. By tuning one of the gratings the next adjacent reflectivity peak can be lined up, which leads to a relatively large tuning step for a small change in control current. Intermediate wavelength steps can be accessed by simultaneously tuning of both gratings. The reflection combs are combined with a multi-mode interference coupler and a phase section, the latter tuning the optical length of the laser. The resulting single peak reflectivity is used as a single feedback wavelength and seeded into the gain medium of the diode laser with the output boosted by a semiconductor optical amplifier (SOA). In total, five current-controlled sections; right and left reflector, phase section, gain section, and SOA, are integrated on the laser chip. The sections have different current ranges, with maximum currents of $7.5 \mathrm{~mA}$ (phase section), $25 \mathrm{~mA}$ (grating reflectors), $100 \mathrm{~mA}$ (gain section), and

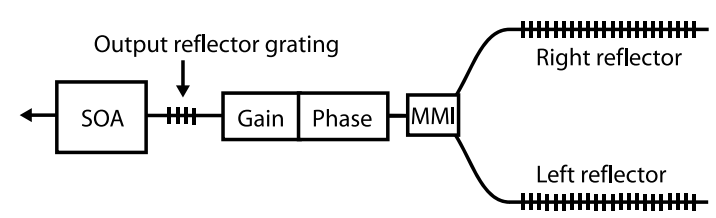

Fig. 1 The MG-Y diode laser sections; two modulated grating reflectors, a multi-mode interference coupler (MMI), a phase section matching the reflection spectra, a gain medium, and a semiconductor optical amplifier (SOA) 
$150 \mathrm{~mA}$ (SOA). The wavelength tuning is achieved by simultaneously changing the currents to the two grating reflectors and the phase section. In contrast to the conventional tuning of a DFB diode laser, this tuning is only associated with a very small accompanied power modulation. However, linear wavelength tuning by the reflection and phase sections is not as straight forward as the tuning of a DFB diode laser. In addition to the demanding synchronized tuning of the three sections, their current-wavelength dependency is not linear, as shown in Fig. 2. The tuning of the reflectors and the phase section is based on changing the amount of carriers in the regions and not on temperature changes, allowing very fast

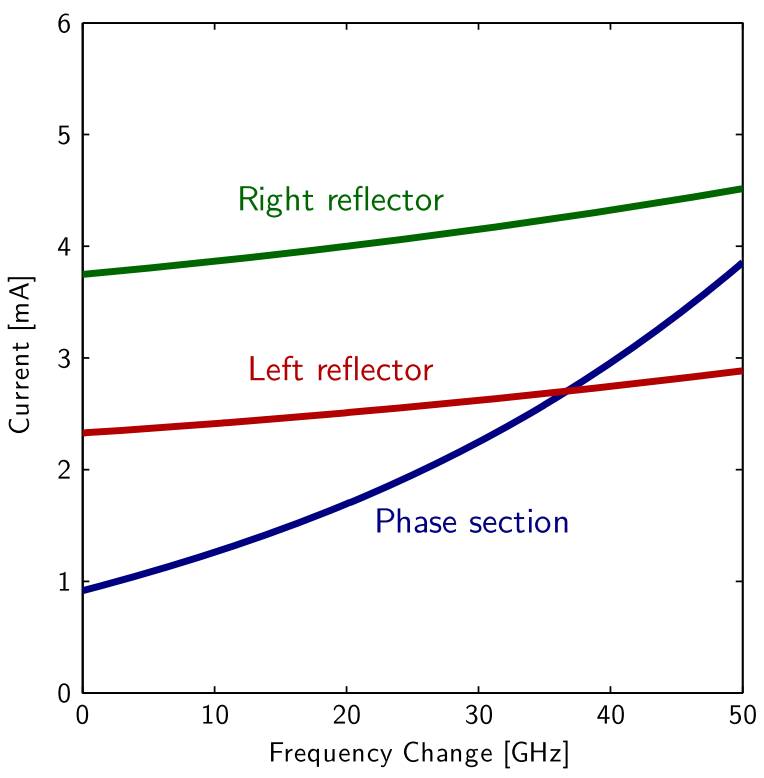

Fig. 2 The dependency of the current to wavelength tuning of the sections of an MG-Y diode laser, for one scanning region tuning. The MG-Y laser itself can be tuned between any two wavelengths in the operating range within $50 \mathrm{~ns}$.

The MG-Y diode laser used was packaged in a similar manner as a typical DFB, and pigtailed to a polarizationmaintaining single-mode fiber. A look-up table for the laser was supplied with specific data on section currents to output frequency calibration. The look-up tables were built into the system software control, with input parameters such as center frequency $(\mathrm{THz})$ as well as scan $(\mathrm{GHz})$ and modulation amplitudes $(\mathrm{GHz})$. The reproducibility of the MG$\mathrm{Y}$ diode laser frequency settings from the manufacturerprovided calibration tables did not exhibit any large deviations from the input set point (less than a $\mathrm{GHz}$ ). This means that within a scan of about $10 \mathrm{GHz}$ (which was used in this system) a desired absorption line, was immediately located. The noted small frequency variation of desired and true operation point is believed to arise from the laser temperature controller. The calibration table is given at a fixed temperature $\left(25^{\circ} \mathrm{C}\right)$ and it is obvious that changes in temperature will induce frequency shifts.

\subsection{Setup}

The multi-species fast gas sensing system consists of a laser system with optics coupling the light into a multi-pass cell, connected with a gas-handling system, see Fig. 3 . The laser is controlled by five specially designed small, low-noise, and low-drift $\left(3 \mathrm{ppm} /{ }^{\circ} \mathrm{C}\right)$ laser drivers, with a digital set point and analog modulation, similar to those described in [16] and [17]. The diode laser controllers operate at different ranges to match the current limits of the laser sections. The noise of the $25 \mathrm{~mA}$ driver was measured to be $20 \mathrm{pA} / \sqrt{\mathrm{Hz}}$, $14 \mathrm{nA}_{\mathrm{RMS}}$ for $30 \mathrm{kHz}$ to $500 \mathrm{kHz}$. The laser chip and

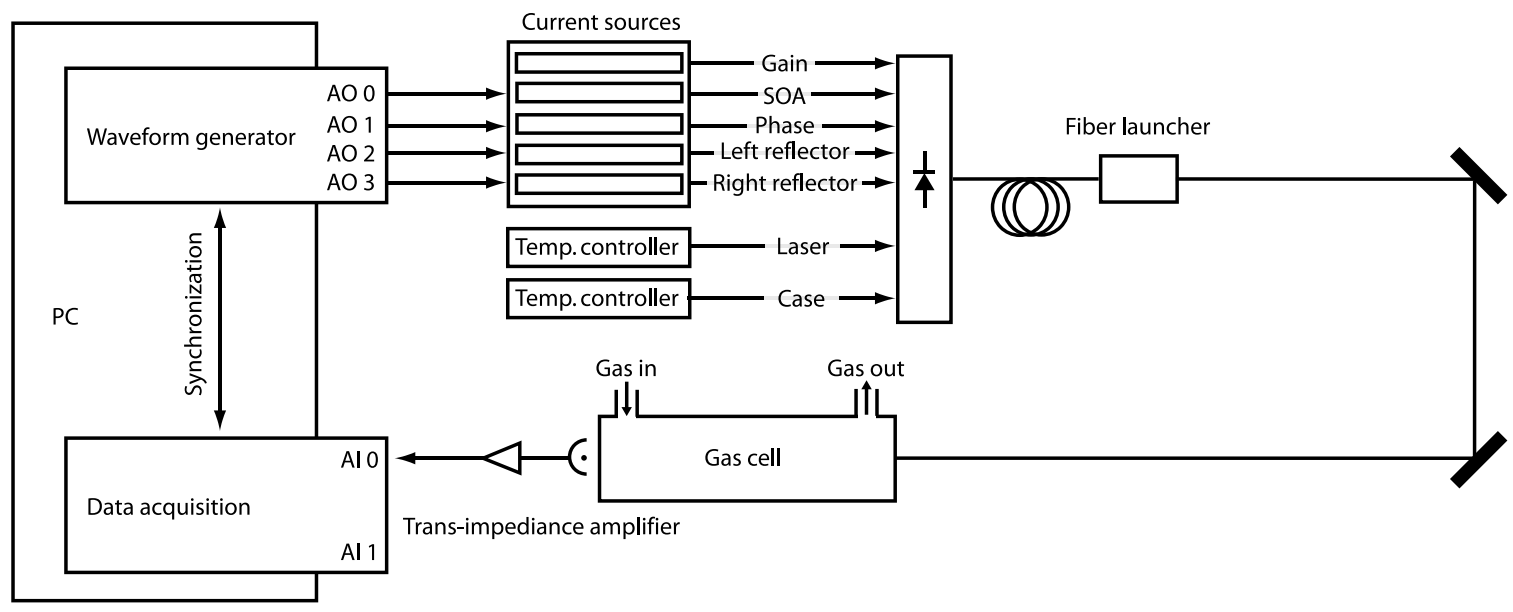

Fig. 3 Schematic of multi-species gas sensing system based on a MG-Y diode laser and digital wavelength modulation spectroscopy. The gas cell is coupled to a gas-handling system providing calibrated gas flow 
laser case are temperature stabilized (TED200 Thorlabs and HTC3000 Wavelength electronics, respectively).

A Herriott type multi-pass cell with a sample volume of 1.41 was used [18]. The cell is optimized for the $3.2-\mu \mathrm{m}$ region, limiting the applicability at $1550 \mathrm{~nm}$ to a lower amount of passes. In this work the $45.6-\mathrm{cm}$ long gas cell was used with 63 passes, resulting in a pathlength of $33.8 \mathrm{~m}$.

The multi-pass cell is connected to a gas-handling system which mixes compressed gas standard mixtures of $\mathrm{CO}, \mathrm{CO}_{2}$, $\mathrm{CH}_{4}$, and $\mathrm{C}_{2} \mathrm{H}_{2}$ with air from a zero-air generator (AAdco Model-737-12A). The mixture concentrations are known to better than $5 \%$ in all cases. A vacuum pump draws the gas through the gas cell with a flow of typically 1-2 SLM (Standard Litres per Minute). The pressure in the cell is held at 50 Torr, yielding absorption lines with a half-widths at half maximum (HWHM) of about 250-350 MHz. Absorption from the free gas in the open path is considered in the fitting model. The transmitted light through the multi-pass cell is detected on an InGaAs photo diode (G8376-05 Hamamatsu) with a photo sensitivity of $0.95 \mathrm{~A} / \mathrm{W}$. The induced photo current is amplified by a low-noise trans-impedance amplifier (DLCPA 200 FEMTO), before being sampled.

To enable fast detection the system is controlled by two synchronized data acquisition (DAQ) cards, responsible for diode laser control and sampling the detected signal. Synchronization of the laser modulation and scan with the detection, which is referred to as coherent sampling, enables many scans to be averaged before the signal is analyzed. The coherent-sampling approach minimizes the necessary computational resources and allows post-processing, such as digital wavelength modulation spectroscopy, dWMS, with Fourier-based signal retrieval (see Sect. 2.4). The DAQ card, which controls the laser drivers, is an arbitrary waveform generation PCI-express card (M2i.6022-exp Spectrum $\mathrm{GmbH})$ with 4 analog (14 bit) and 8 digital outputs with a sampling frequency of $60 \mathrm{MS} / \mathrm{s}$. The detected signal is sampled with a digitizer PCI-express card (M2i.4651-exp Spectrum $\mathrm{GmbH}$ ) with 4 analog inputs (16 bit), and a $3 \mathrm{MS} / \mathrm{s}$ sampling rate, and as already stated synchronized with the output card. The five controllers, specially designed to control the five diode laser sections, are operated through a digital set point and an analog modulation.

The system has a very low drift (10 MHz), due to small noise at low frequencies and a larger noise at higher frequencies leading to a broad linewidth (70 MHz-HWHM), higher than specified by the manufacturer and previous literature values [6]. When averaged, higher frequency noise will not affect accurate measurements of the center frequency. The center frequency is mainly determined by the digital set point, which is not affected by the analog noise pick-up in the cable, as it is sent digitally to the driver. Although the root causes for the broadened linewidth has yet to be confirmed, one possible cause could be offsets from optimally matching currents for the different laser sections. The relative value of the currents to the different section was chosen in accordance with the factory calibrated look-up tables, but was not varied from these value to minimize the noise. Noise pick-up of the cables from the separate diode laser drivers are suspected as another one. The characteristics of the noise source of the laser could not be further studied in detail, since they could not be isolated and identified. The noise specification of each laser driver does not support the larger measured linewidth. This may be alleviated with the use of five drivers incorporated into a single unit, similar as the driver provided by the manufacturer which is designed for telecom applications. Nevertheless, aside from an instrumental function that must be incorporated when deducing linecenter absorbance from direct absorption measurements, this does not play a large role in affecting the results in the presented study. Laser lineshape and width determinations were acquired by beating the MG-Y laser output with a tunable external cavity diode laser with a narrow linewidth, on a fast photodetector. Incorporating the instrumental function of $70 \mathrm{MHz}$ linewidth into the fitting of a theoretical basefunction (discussed later) based on HITRAN data yielded a very good fit to true absorption lines.

\subsection{Time multiplexing}

Multi-species detection is implemented by time multiplexing consecutive scans over each absorption line segment, with discrete jumps in between, see Fig. 4. Each scan segment of $10 \mathrm{GHz}$ only constitutes $0.2 \%$ of the tuning region of $4510 \mathrm{GHz}(191,561-196,071 \mathrm{GHz})$ of the laser, and thus only appears as horizontal lines in the graph. Each scan segment consists of a slower ramp frequency and a higher mod-
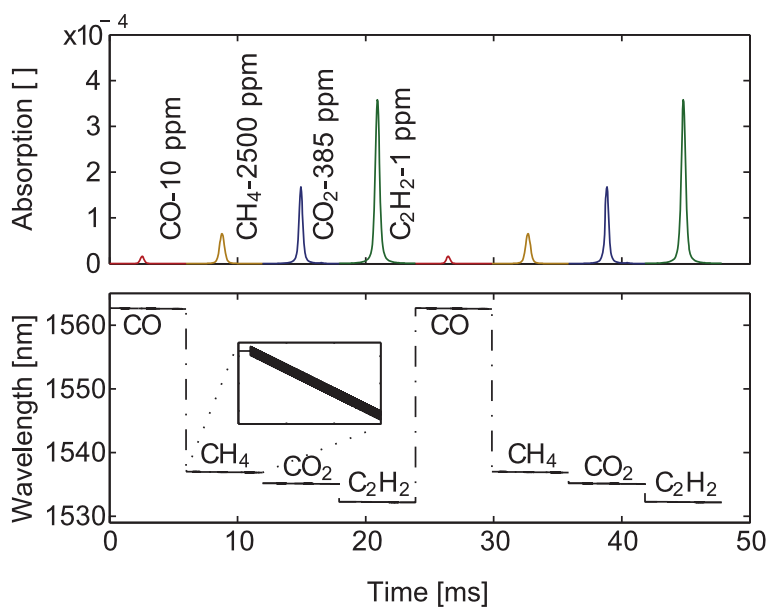

Fig. 4 Scan procedure of multi-species sensing with the MG-Y diode laser. The laser wavelength is swept over an absorption line followed by a large discrete jump to another absorption line. The small scanned region over the absorption appear as horizontal lines compared to the discrete jumps due to the difference in scale. An enlarging of the scan is presented in the inset 
ulation (see the inset of Fig. 4). The sweep time of one scan segment is $5.5 \mathrm{~ms}$ following a settling time of $0.5 \mathrm{~ms}$, required by the electronics. Repeated scans over each absorption region are possible without active wavelength locking due to the MG-Y diode laser's stable operating conditions, which means that the operating point of the laser hits the exact same frequency (within $10 \mathrm{MHz}$ ) in the next scan sequence.

Several interesting gases absorb within the operating range $(1529 \mathrm{~nm}-1565 \mathrm{~nm})$ of the MG-Y diode laser. In this work the gases $\mathrm{CO}, \mathrm{CO}_{2}, \mathrm{CH}_{4}$, and $\mathrm{C}_{2} \mathrm{H}_{2}$ with absorption lines ranging from $1532 \mathrm{~nm}$ to $1563 \mathrm{~nm}$ were studied (see Fig. 5). The scanned regions are indicated in black and enlarged in the insets of the graphs.

For each individual scan, both the data controlling the diode laser and the acquired data fulfill the criteria of coherent sampling, i.e. there is a specific relation between the sampling and modulation frequency resulting in an exact number of sampled cycles, preferably a prime number. For this work the sampled data from one gas-absorption scan
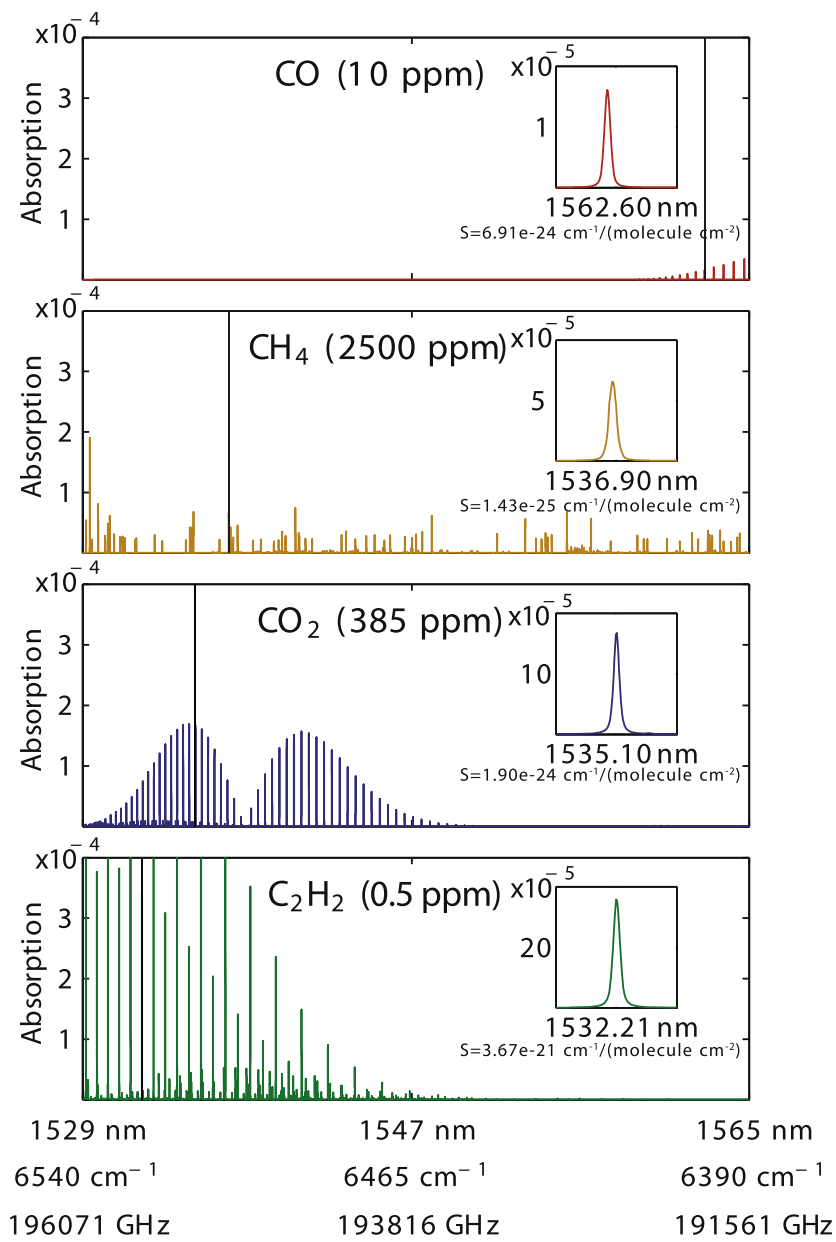

Fig. 5 Absorption lines of interesting gases within the MG-Y diode laser operating range. The black vertical line indicates the scanned region and is enlarged in the inset of each graph. Data are simulated using the HITRAN data base [19] was 16384 samples long and contained 821 cycles. These values correspond to a modulation frequency of approximately $150 \mathrm{kHz}$.

\subsection{Signal processing-harmonic detection}

High-sensitivity TDLAS spectrometry in many cases is aided by the use of modulation techniques. To enable fast detection, coherent sampling and digital wavelength modulation spectroscopy, dWMS, using Fourier signal filtering, are employed. Fourier-based signal retrieval is in principle equivalent to lock-in based signal retrieval, but much more flexible. A detailed description is outside the scope of this paper and can be found elsewhere [20-22]. Briefly, a complex time representation of the desired WMS harmonic is retrieved by selecting only the positive harmonic component with a window function in the Fourier plane (see Fig. 6). The harmonic signal of interest (2nd harmonic in this case) is shifted to zero frequency and down-sampled to enable real-time fast data processing, by simply truncating the data to the low frequency Fourier data component. An inverse Fourier transform creates a complex time representation of the harmonic signal. This means that a pure sine signal at the harmonic frequency and with a fixed amplitude will appear as a point in the complex plane, whose absolute value corresponds to the amplitude of the original signal and the angle represents its phase. By projecting the complex function to the real axis and only studying the real component of the signal, the traditional WMS signal obtained with a lock-in amplifier can be retrieved (see Fig. 6). All harmonics are available using this procedure since the raw data of the signals are used, which is made possible due to the coherent sampling, which in turn allows averaging of several scans.

The measured absorption is extracted by fitting the measured absorption signal $\bar{v}_{s}^{*}(v)$, to a model, (1), including a

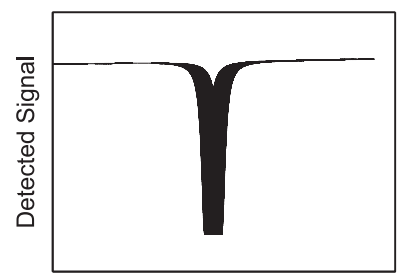

Time

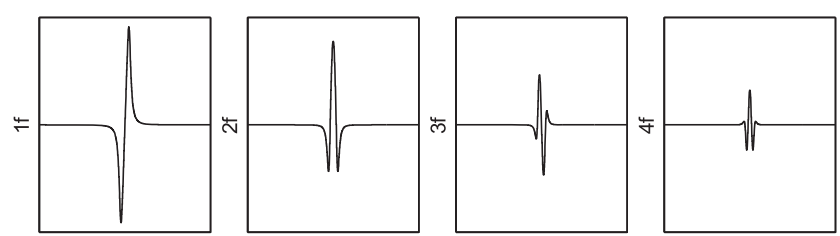

Fig. 6 Simulation data of WMS generation and extraction. The non-linear response at the absorption (top left) creates overtones, present int he FFT (top right). Filtering of a harmonic by a window function, red in FFT, yield WMS signals (bottom) 
background basefunction, $\bar{v}_{\mathrm{bkg}}^{*}(\nu)$, and an absorption imprint, $\bar{v}_{\mathrm{abs}}^{*}(\nu)$, which is either theoretically calculated or measured with a known high concentration gas mixture. The theoretical Voigt profile is calculated using parameters from HITRAN and takes the laser linewidth into account using a Gaussian profile. The background basefunction includes the gas-absorption contribution from the open path. The fitting parameter, $a_{n}^{*}, b^{*}$, and $c^{*}$, are complex constants, where the absolute value of $c^{*}$ times the concentration of the high concentration gas mixture yields the concentration of the studied gas.

$\bar{v}_{s}^{*}(v) \simeq \bar{v}_{\mathrm{bkg}}^{*}(v)+c^{*} \cdot \bar{v}_{\mathrm{abs}}^{*}(v)$,

$\bar{v}_{s}^{*}(v) \simeq \sum_{n=0}^{N} a_{n}^{*} \cdot v^{n}+b^{*} \cdot \bar{v}_{\mathrm{za}}^{*}(v)+c^{*} \cdot \bar{v}_{\mathrm{abs}}^{*}(v)$.

The background basefunction consists of an absorption-free pattern, $\bar{v}_{\mathrm{za}}^{*}(v)$, measured while flushing zero air into the gas cell, and a polynomial, $\sum a_{n}^{*} \cdot v^{n}$, with $N=3$, accounting for offset, linear and quadratic background structures during each scan. Such background acquisition removes any optical noise that may arise from interference fringes from a variety of sources and was acquired with a repetition frequency determined from Allan-variance measurements discussed in the next section. A routine that acquires this zero-air component is incorporated into the measuring procedure, and controlled by the setup software.

\section{Measurements}

\subsection{System performance}

The system performance was tested by studying the Allan variance under a variety of cell input concentrations. We first show results when zero air was input into the multi-pass cell. All results are at the $1 \sigma$ level. Figure 7 shows the results of fitting a high and known absorption concentration from an $\mathrm{C}_{2} \mathrm{H}_{2}$ spectrum to zero-air spectra.

Figure 7, and shows how the different harmonic WMS signals yield different sensitivities. As shown, the system has a minimum detection limit of $1.6 \times 10^{-7}$ absorbance (base e) or absorption fraction for the $1 f$-WMS signal and $2.0 \times 10^{-7}$ for the $2 f$-WMS signal, with an averaging time of $20 \mathrm{~s}$. Corresponding numbers for $55 \mathrm{~ms}$-average time are $2.0 \times 10^{-6}$ and $3.7 \times 10^{-6}$, respectively. At 1 second of averaging, the corresponding numbers are $5 \times 10^{-7}$ and $9 \times 10^{-7}$, respectively. The decrease in sensitivity at higher harmonics is to be expected due to the lower signal values $[23,24]$. Although the $1 f$-WMS signal provides the strongest WMS signal, it is often not selected due to the unwanted RAM offset and the higher sensitivity to optical noise. Although the prior issue is not a concern for the MG-Y

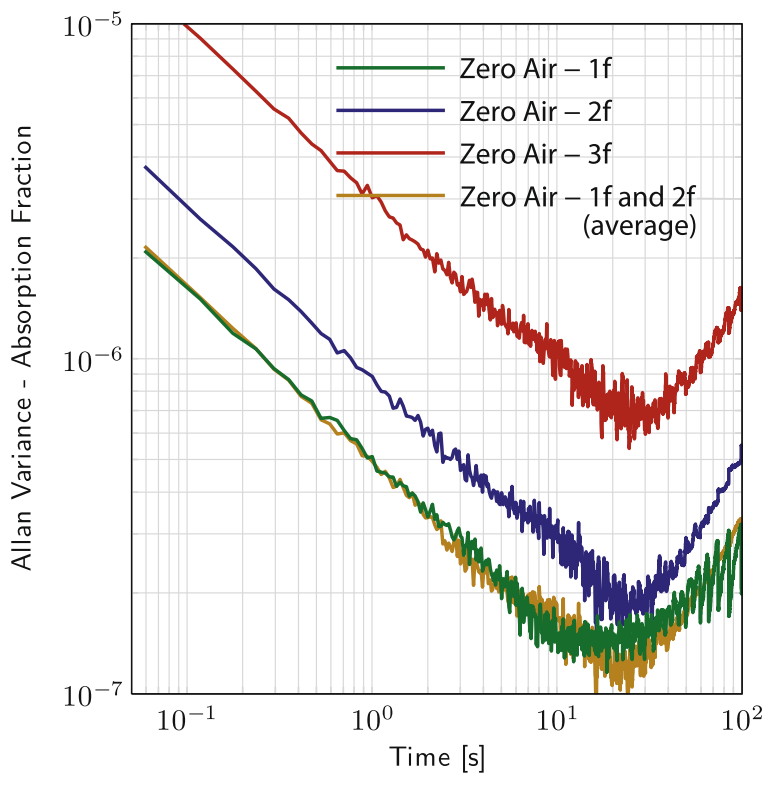

Fig. 7 System performance investigation through zero-air fitting of an absorption of $\mathrm{C}_{2} \mathrm{H}_{2}(1531.6 \mathrm{~nm})$ for different WMS harmonics

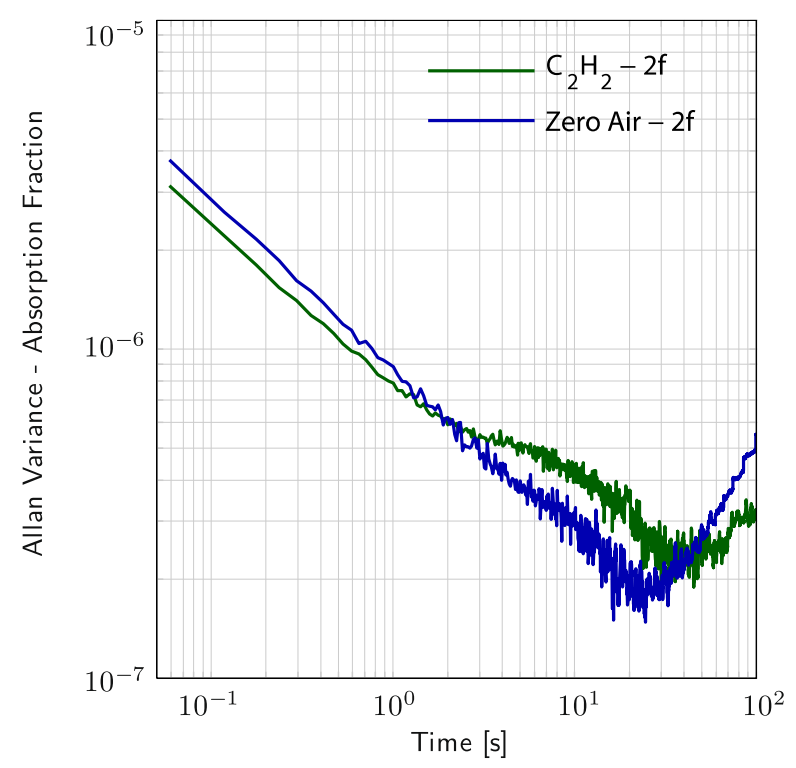

Fig. 8 Comparison of system performance using based on the $2 f$ WMS signal of an absorption signal $\left(\mathrm{C}_{2} \mathrm{H}_{2}\right.$ at $\left.1531.6 \mathrm{~nm}\right)$ and the present background (zero air)

setup the latter effect can be seen as drifts in the Allan variance, i.e. turning up of the curve. The corresponding knee of the $2 f$ signal appears later. It is interesting to note that the best sensitivity with longest stability is achieved by using the average of both the $1 f$ and $2 f$ signals. However, due to the drift sensitivity of the $1 f$ signal and as well as to provide data which can be compared to other reported data in the literature, the gas sensing performance studied in this work is based on $2 f$ WMS data. 


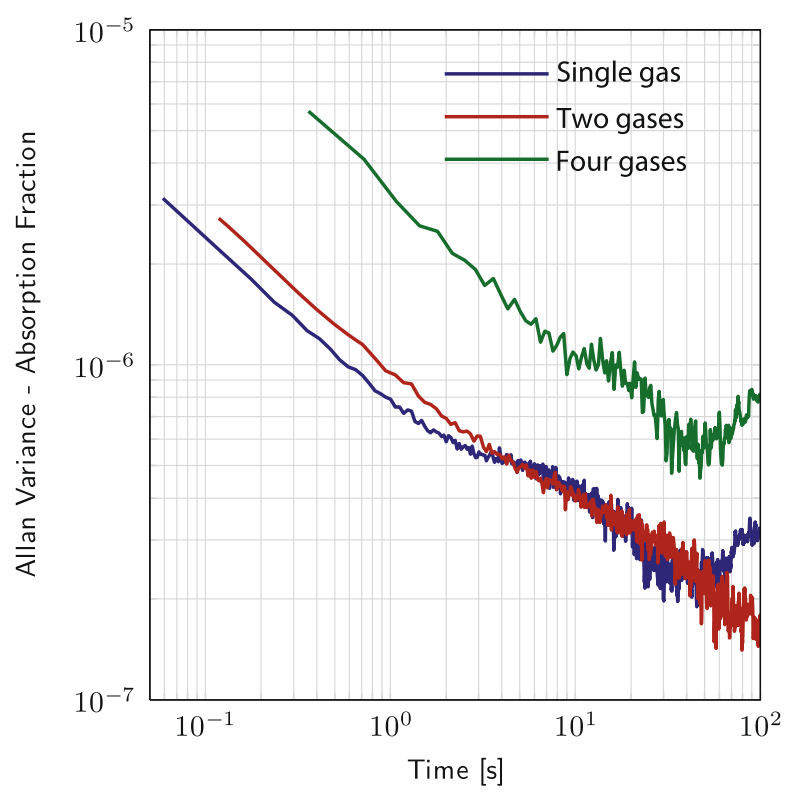

Fig. 9 Comparison between single gas, two gas, and multiple gas sensing. The data are measured on $\mathrm{C}_{2} \mathrm{H}_{2}$ alone, with simultaneous monitoring of $\mathrm{CO}$ for the two gas case, and $\mathrm{C}_{2} \mathrm{H}_{2}, \mathrm{CO}, \mathrm{CO}_{2}$, and $\mathrm{CH}_{4}$ for the multi-species case

Figure 8 plots the Allan variance for both the zero air and for measurements acquired using $50 \mathrm{ppb}$ concentration of $\mathrm{C}_{2} \mathrm{H}_{2}$, which produces an absorbance of about $1.1 \times 10^{-4}$. As shown, the $2 f$ performance of the latter is slightly better than the former at shorter averaging times, which we believe originates from the fact that the fitted absorption line better locks to the absorption imprint of $\mathrm{C}_{2} \mathrm{H}_{2}$ with the correct complex orientation in contrast to when no absorption (zero air) is present. The reverse behavior is noted at larger average times, perhaps due to imprecision in the $\mathrm{C}_{2} \mathrm{H}_{2}$ gas mixture introduction or an unknown system drift. In any event

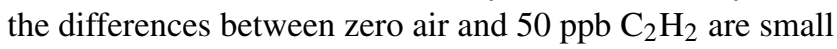
and at 1 second integration are very close to the $2 f$ value obtained at 1 second in Fig. 7.

\subsection{Multi-species detection}

The effect on the absorbance sensitivity as multiple gases are sensed was investigated by analyzing the Allan variance for $\mathrm{C}_{2} \mathrm{H}_{2}$ when detected alone, together with an additional gas and with three additional gases. In all cases we study the effect on $\mathrm{C}_{2} \mathrm{H}_{2}$ and assess the results of the time multiplexing of additional gases, which reduces the integration time on the $\mathrm{C}_{2} \mathrm{H}_{2}$ feature. These results are shown in Fig. 9. The time multiplexing when adding additional scan segments to access additional gases degrades the sensitivity due to the reduction in averaging time for each gas. At $1 \mathrm{sec}-$ ond Fig. 9 shows an approximate difference of $\sqrt{2}$ for sensitivity difference in the detection of $\mathrm{C}_{2} \mathrm{H}_{2}$ alone compared to adding a second gas. When sensing 4 gases simultaneously,

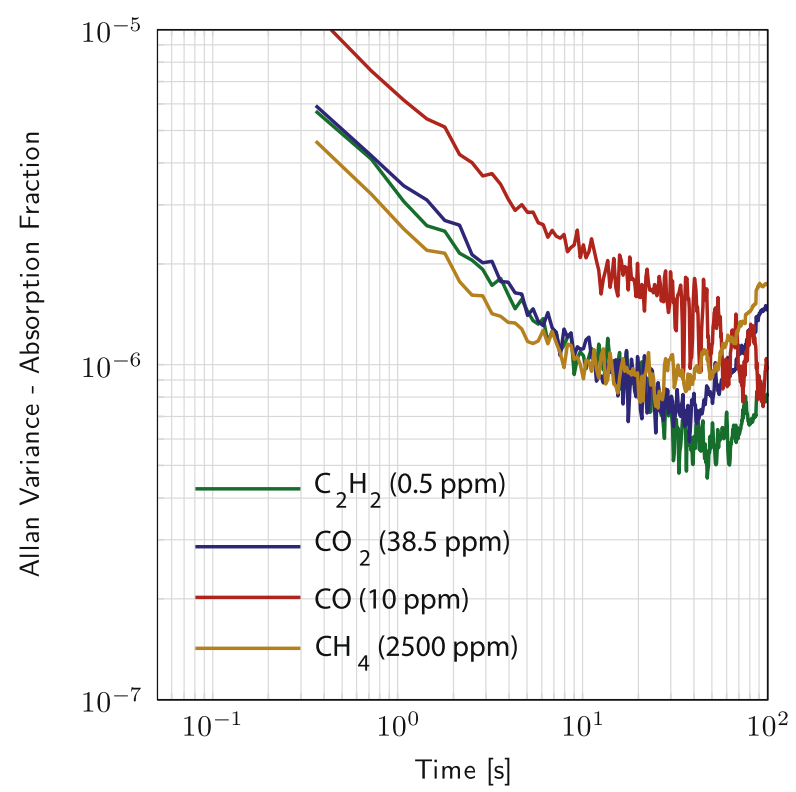

Fig. 10 The Allan variance of four gases sensed by sequentially scanning of individual lines using a MG-Y diode laser

however, the degradation compared to single-gas sensing is $\sim 3.8$, which is higher than the factor of $\sim 2$ expected.

When monitoring four gases the system shows a best detection sensitivity of $6 \times 10^{-7}$ in 40 seconds for $\mathrm{C}_{2} \mathrm{H}_{2}$. The 1 second averaging time yields a detection sensitivity of $3 \times 10^{-6}$. Figure 10 plots the retrieved results for the four different gases when acquired using the sequential scan segment approach. The results for $\mathrm{C}_{2} \mathrm{H}_{2}, \mathrm{CH}_{4}$, and $\mathrm{CO}_{2}$ are nearly the same but the results for $\mathrm{CO}$ are about a factor of 2 worse. In addition to being the smallest absorption feature (see Fig. 5), the CO fitting model was not ideal in this circumstance. In contrast to normal zero-air generator behavior, where $\mathrm{CO}$ is completely removed, we observed residual $\mathrm{CO}$ during background acquisition in these runs.

\section{Discussion}

Absorption spectroscopy with diode lasers have long been limited by the tunability of the laser source. Multiplexing of several diode laser are possible but is costly and more complex. The use of an external cavity allow a large tunability but is limited in the tuning performance, i.e. repeatable controlled tuning. By contrast, the MG-Y diode laser discussed in this study not only provides extended tunability but also precise controllability, which allows multi-species detection with a single laser. We have employed multi-species detection by time multiplexing small scan segments in wavelength over individual absorption lines and made discrete jumps between these. Sequentially jumping between regions is possible due to the repeatability of the laser output wavelength for the same settings. It is worth noting that although 
the laser can access features separated by over $30 \mathrm{~nm}$, the set point is still precise to within $10 \mathrm{MHz}$. Thus the MG-Y diode laser exhibits a very large dynamic range. The system design is flexible, allowing the user to build scan sequences, with varying number of scans and sweep settings, and loading the new parameters within seconds. Through the manufacturer-provided look-up table the output wavelength could be predicted, within $1 \mathrm{GHz}$, allowing the possibility to quickly identify an interesting region and load it into the system. In addition, the $10 \mathrm{MHz}$ repeatability obviates the need for separate wavelength locking cells and detectors.

The residual amplitude modulation, RAM, present when modulating most diode lasers, such as DFB and VCSEL units, is very small in the MG-Y diode laser, making it a very attractive laser source that does not require an additional detector for capturing and removing the resulting optical structure. Furthermore, the MG-Y diode laser is mounted with a semiconductor optical amplifier (SOA) on the chip allowing for amplitude modulation of the laser. This can be applied to suppress any small residual RAM of the MG-Y diode laser, eliminate stable fringes, but also to amplitude modulate (AM) the diode laser. Work has been carried out to distinguishing the AM WMS signal from the FM induced WMS signal to study the absorption lineshape [25]. Furthermore, the SOA can also be used for studies of the effect of RAM on various optical components.

For ultra high-sensitivity absorption spectroscopy one would like to utilize the strong absorptions available in the mid infrared. To transfer the tuning properties of the MG-Y laser to the mid infrared two tunable frequency's could be mixed in a periodically poled lithium niobate (PPLN) crystal to generate the difference frequency. If both sources are tuned simultaneously the PPLN temperature does not have to be changed during the tuning. The MG-Y laser would be very suitable to use as one of the tunable sources. For example could two lasers, one tunable between 1027-1106 nm, and the other one tunable between $1532-1563 \mathrm{~nm}$, generate light in the whole range between $3000-3800 \mathrm{~nm}$, for a PPLN with a poling period of $29.8 \mu \mathrm{m}$ and at a temperature of $41^{\circ} \mathrm{C}$. This tuning would not require changing the PPLN temperature.

Within the environmental field, the MG-Y diode laser has the potential to provide many interesting applications. For example, at the National Center for Atmospheric Research a major focus is on measuring many trace atmospheric gases simultaneously from airborne platforms. The limited space and harsh environmental conditions present on such platforms, where one often experience large cabin temperature and pressure swings along with vibrations, demands robust, reliable, and small instruments with autonomous operation. Although the present MG-Y laser discussed in this study adds some complexity by requiring five low-noise laser driver sources, the high sensitivity, extreme versatility, and flexibility demonstrated here provides the possibility of building such a small, robust, and flexible multi-species TDLAS instrument.

In summary we present fast, high-sensitivity multispecies detection in the near-IR by sequentially scanning individual absorption lines followed by discrete jumps using a widely tunable MG-Y diode laser. The MG-Y diode laser employs the Vernier effect, allowing it to be reliably controlled to any wavelength within the tuning range $(1529 \mathrm{~nm}-$ $1565 \mathrm{~nm}$ ), and does not exhibit large unwanted RAM. One second measurements resulted in a $1 \sigma$ absorbance sensitivity ranging between $5-8 \times 10^{-7}$ when sensing only a single gas and $3 \times 10^{-6}$ when detecting four gases employing fast sequential scanning over a $30 \mathrm{~nm}$ range. The flexibility of the MG-Y diode laser together with its reliable performance makes it an attractive TDLAS laser source for many applications. The present laboratory demonstration of highly sensitive and fast detection of four atmospherically important trace gases between 1529-1562 nm using an MG-Y laser illustrates the potential of such a laser source in further developing robust, widely tunable diode laser based instruments for multi-species detection.

Acknowledgements Support by Sune Svanberg, Lund University, Sweden is gratefully acknowledged. The authors also acknowledge James Walega for the assistance with the gas-handling system and Terry Hock, Eric Loew, and Mike Strong for their valuable input regarding electronic design.

The National Center for Atmospheric Research is sponsored by the National Science Foundation and operated by the University Corporation for Atmospheric Research.

This work was supported in part by the Swedish Natural Science Research Council, the VINNOVA PIEp programme, and NCAR's Advanced Study programme.

\section{References}

1. P. Werle, Spectrochim. Acta A 54, 197 (1998)

2. P. Werle, F. Slemr, K. Maurer, R. Kormann, R. Mücke, B. Jänker, Opt. Lasers Eng. 37, 101 (2002)

3. A. Fried, D. Richter, in Analytical Techniques for Atmospheric Measurement, ed. by D.E. Heard (Wiley, New York, 2007)

4. M. Lackner, Rev. Chem. Eng. 23, 65 (2007)

5. P. Martin, Chem. Soc. Rev. 31, 201 (2002)

6. V. Weldon, D. McInerney, R. Phelan, M. Lynch, J. Donegan, Spectrochim. Acta A 63, 1013 (2006)

7. V. Jayaraman, Z.M. Chuang, L.A. Coldren, IEEE J. Quantum Electron. 29, 1824 (1993)

8. T.L. Koch, U. Koren, J. Lightwave Technol. 8, 274 (1990)

9. F. Koyama, J. Lightwave Technol. 24, 4502 (2006)

10. B.G. Lee, M.A. Belkin, R. Audet, J. MacArthur, L. Diehl, C. Pflugl, F. Capasso, D.C. Oakley, D. Chapman, A. Napoleone, D. Bour, S. Corzine, G. Hofler, J. Faist, Appl. Phys. Lett. 91, 231101 (2007)

11. M. Lackner, M. Schwarzott, F. Winter, B. Kogel, S. Jatta, H. Halbritter, P. Meissner, Opt. Lett. 31, 3170 (2006)

12. W. Zeller, M. Legge, J. Seufert, R. Werner, M. Fischer, J. Koeth, Appl. Opt. B 48, B51 (2009) 
13. M. Chacinski, M. Isaksson, R. Schatz, IEEE Photonics Technol. Lett. 17, 1157 (2005)

14. R. Phelan, M. Lynch, J.F. Donegan, V. Weldon, Appl. Opt. 44, 5824 (2005)

15. P. Cancio, P. Zeppini, P. De Natale, S. Taccheo, P. Laporta, Appl. Phys. B 70, 763 (2000)

16. K.G. Libbrecht, J.L. Hall, Rev. Sci. Instrum. 64, 2133 (1993)

17. C. Erickson, M. Van Zijll, G. Doermann, D. Durfee, Rev. Sci. Instrum. 79, 073107 (2008)

18. D. Richter, S. Spuler, in Conference on Field Applications in Industry and Research (2009)

19. L.S. Rothman, I.E. Gordon, A. Barbe, D.C. Benner, P.E. Bernath, M. Birk, V. Boudon, L.R. Brown, A. Campargue, J.P. Champion, K. Chance, L.H. Coudert, V. Dana, V.M. Devi, S. Fally, J.M. Flaud, R.R. Gamache, A. Goldman, D. Jacquemart, I. Kleiner, N. Lacome, W.J. Lafferty, J.Y. Mandin, S.T. Massie, S.N.
Mikhailenko, C.E. Miller, N. Moazzen-Ahmadi, O.V. Naumenko, A.V. Nikitin, J. Orphal, V.I. Perevalov, A. Perrin, A. Predoi-Cross, C.P. Rinsland, M. Rotger, M. Simeckova, M.A.H. Smith, K. Sung, S.A. Tashkun, J. Tennyson, R.A. Toth, A.C. Vandaele, J. Vander Auwera, J. Quant. Spectrosc. Radiat. Transf. 110, 533 (2009)

20. T. Fernholz, H. Teichert, V. Ebert, Appl. Phys. B 75, 229 (2002)

21. M. Andersson, L. Persson, T. Svensson, S. Svanberg, Rev. Sci. Instrum. 78, 113107 (2007)

22. T. Svensson, M. Andersson, L. Rippe, S. Svanberg, S. AnderssonEngels, J. Johansson, S. Folestad, Appl. Phys. B 90, 345 (2008)

23. J. Reid, D. Labrie, Appl. Phys. B 26, 203 (1981)

24. P. Kluczynski, J. Gustafsson, A.M. Lindberg, O. Axner, Spectrochim. Acta B 56, 1277 (2001)

25. K. Duffin, A.J. McGettrick, W. Johnstone, G. Stewart, D.G. Moodie, J. Lightwave Technol. 25, 3114 (2007) 